\title{
О ПОДАВЛЕНИИ НИЗКОЧАСТОТНЫХ ГАРМОНИК В ТРЕХФАЗНОМ ВЫПРЯМИТЕЛЕ С ПОМОЩЬЮ ЗАКОНА УПРАВЛЕНИЯ ТИРИСТОРАМИ
}

\author{
(Представил Н. Эпик)
}

Несимметричность питающей сети, а также несимметричность системы управляющих импульсов обусловливают появление на выходе управляемого выпрямителя спектра с частотами

$$
\omega=n \omega_{0} ; \quad n=1, \ldots, 5 \text {, }
$$

где $\omega_{0}-$ частота сети.

Оценке уровня данных гармоник посвящено много работ. Однако формулы, полученные в этих работах, очень громоздки [ [ $\left.{ }^{1}\right]$ и выведены для частных случаев $\left[{ }^{2,3}\right]$. Поэтому вопрос о возможностях подавления низкочастотных гармоник подходящим управлением момента включения отдельных тиристоров в общем случае остался открытым.

В данной работе поставлена цель вывести для низкочастотных гармоник простые соотношения как основы решения выше сформулированной проблемы. Исследование проведено для трехфазного мостового выпрямителя, работающего на линейную нагрузку с достаточно большой индуктивностью, чтобы обеспечить режим непрерывного тока. Тиристоры рассмотрены как идеальные ключи. Формулы даны для напряжений.

Обычно при нахождении спектра выходного напряжения выпрямителя сначала суммируются временные кривые входных напряжений и затем вычисляются коэффициенты Фурье для суммарной кривой. Свойство линейности преобразования Фурье дает нам возможность использовать принцип наложения: сначала вычислить спектры, даваемые отдельными фазами, и потом их суммировать. При суммировании необходимо учитывать свойство смещения преобразования Фурье, которое можно сформулировать следующим образом: сдвиг фазы на угол $\varphi$ в исследуемой периодической кривой приводит к сдвигу фазы на $n \varphi$ в $n$-й гармонике $\left[{ }^{4}\right]$. Сдвиги фаз низкочастотных $(n=1, \ldots, 5)$ гармоник и расположение их в векторной форме в случае трехфазной сети приведены в таблице, где через $a$ обозначен комплексной оператор $a=\mathrm{e}^{j 2 \pi / 3}$. Из таблицы видно, что гармоники, кратные трем, складываются в фазе, отчего уровень их на выходе существенно повышается. Для подавления нечетных гармоник необходимо выбирать закон управления таким образом, чтобы в каждой фазе углы отпирания в обоих полупериодах были одинаковыми. Последнее требование вполне реализуемо [5].

Проанализируем генерирование второй и четвертой гармоник при 


\begin{tabular}{|c|c|c|c|c|}
\hline \multirow{2}{*}{$\begin{array}{l}\text { Номер } \\
\text { гармо- } \\
\text { ники }\end{array}$} & \multicolumn{3}{|c|}{ Фаза } & \multirow{2}{*}{ Векторы } \\
\hline & $A$ & B & C & \\
\hline 1 & 1 & $a^{2}$ & $a$ & \\
\hline 2 & 1 & $a$ & $a^{2}$ & \\
\hline 3 & 1 & 1 & 1 . & \\
\hline 4 & 1 & $a^{2}$ & $a$ & \\
\hline 5 & 1 & $a$ & $a^{2}$ & \\
\hline 6 & 1 & 1 & 1 & $\uparrow \uparrow \uparrow$ \\
\hline
\end{tabular}

отсутствии нечетных гармоник. Рассмотрим трехфазную сеть с фазными напряжениями

$$
U_{A}=V_{A}, \quad U_{B}=a^{2} V_{B}, \quad U_{C}=a V_{C} .
$$

Отклонения модуля и фазы напряжения отдельных фаз сети от симметричного будем обозначать соотвегственно через $\Delta$ и $\psi$. Для фазы $A$, например, можем написать

$$
V_{A}=\left(V+\Delta_{A}\right) \mathrm{e}^{j \psi_{A}} .
$$

Вторая гармоника, генерируемая фазой $A$ на нагрузке, дается выражением

$$
z_{A 2}=\left(V_{A} / \pi\right) \int_{\varphi+\chi_{A}}^{2 \pi / 3+\varphi+\chi_{B}} j\left(\mathrm{e}^{-j\left(x+\psi_{A}\right)}-\mathrm{e}^{j\left(x+\psi_{A}\right)}\right) \mathrm{e}^{-j 2 x} d x
$$

где $\varphi-$ угол включения тиристора, равный $30^{\circ}+\alpha(\alpha-$ угол управления); $\chi$ - отклонение угла $\varphi$ в соответствующей фазе.

После интегрирования и использования обозначений

$$
F=\mathrm{e}^{-j \varphi}, \quad H_{A}=\mathrm{e}^{-j \chi_{A}}, \quad P_{A}=\mathrm{e}^{-j \psi_{A}}
$$

получаем

$$
z_{A 2}=V_{A} F\left(F^{2} P_{A}\left(H_{A}^{3}-H_{B}^{3}\right) / 3-\bar{P}_{A}\left(H_{A}-a^{2} H_{B}\right)\right) / \pi .
$$

Переменные $H$ означают, что мы рассматриваем систему управляемых импульсов как трехфазную систему единичных векторов. В дальнейшем будем использовать разложения этой системы на симметричные компоненты.

Вторую гармонику выпрямленного напряжения получаем, пользуясь данными таблицы:

$$
z_{2}=z_{A 2}+a z_{B 2}+a^{2} z_{C 2}
$$

или

$$
z_{2}=F\left[V_{A}\left(P_{A} F^{2}\left(H_{A}^{3}-H_{B}^{3}\right) / 3+\bar{P}_{A}\left(a^{2} H_{B}-H_{A}\right)\right)+\right.
$$




$$
\begin{aligned}
& +a V_{B}\left(P_{B} F^{2}\left(H_{B}^{3}-H_{C}^{3}\right) / 3+\bar{P}_{B}\left(a_{2} H_{C}-H_{B}\right)\right)+ \\
& \left.+a^{2} V_{C}\left(P_{C} F^{2}\left(H_{C}^{3}-H_{A}^{3}\right) / 3+\bar{P}_{C}\left(a^{2} H_{A}-H_{C}\right)\right)\right] / \pi .
\end{aligned}
$$

Учитывая, что отклонения сети и углов включения тиристоров от симметричного невелики, можем использовать следующие соотношения для получения первого приближения:

$$
H_{A} \approx 1-j \chi_{A}, \quad P_{A} \approx 1-j \psi_{A} .
$$

Подставляя (9) в выражение (8) и пренебрегая малыми, выше первого порядка, т. е. содержащими члены типа $\chi \Delta, \chi \psi, \Delta \psi$, получаем

$$
z_{2}=3 F\left[\left(a^{2}-1\right) U_{A 2} f\left(F^{2}\left(1-a^{2}\right)+a-1\right) V H_{A 2}\right] / \pi,
$$

где

$U_{A 2}=\left(U_{A}+a^{2} U_{B}+a U_{C}\right) / 3 \approx\left(\Delta_{A}+a \Delta_{B}+a^{2} \Delta_{C}+j\left(\psi_{A}+a \psi_{B}+a^{2} \psi_{C}\right)\right) / 3$,

$H_{A 2}=\left(H_{A}+a H_{B}+a^{2} H_{C}\right) / 3 \approx-j\left(\chi_{A}+a \chi_{B}+a^{2} \chi_{C}\right) / 3$.

Если тиристоры включаются симметрично, то $H_{A 2}=0$ и для амплитуды второй гармоники имеем простую формулу

$$
\left|z_{2}\right|=3 \sqrt{3} \pi^{-1}\left|U_{A 2}\right|
$$

Отметим, что коэффициент $3 \sqrt{3} / \pi \approx 1,65$ получается по отношению к напряжению фазы. Данный результат хорошо согласуется с результатами измерений в $\left[{ }^{6}\right]$. В случае подавления второй гармоники $z_{2}=0$ и углы включения тиристоров получаются из следующего соотношения:

$$
H_{A 2}=\left(\left(F^{2}+a\right)^{-1} U_{A 2}\right) / V .
$$

Модуль множителя перед $U_{A 2}$ зависит от угла управления (см. рисунок). При малых углах управления подавление второй гармоники выбором углов включения тиристоров реализовать невозможно, так как влияние асимметрии тиристоров на вторую гармонику намного меньше влияния асимметрии сети.

Выведем по аналогии выражения и для четвертой гармоники. Для фазы $A$ можем записать

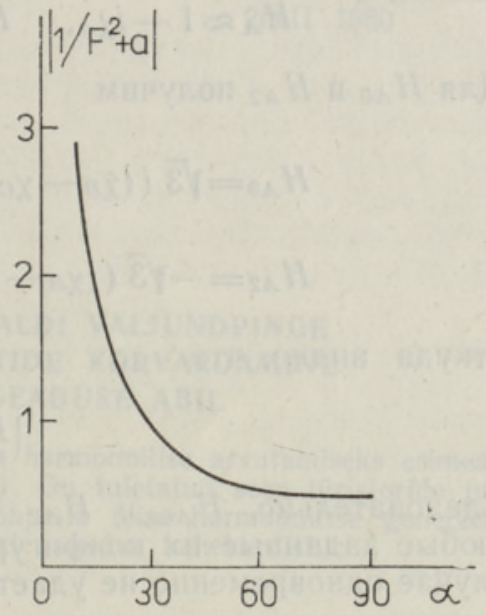

$$
z_{A 4}=\left(V_{A} / \pi\right) \int_{\varphi+\chi_{A}}^{2 \pi / 3+\varphi+\chi_{B}} j\left(\mathrm{e}^{-j\left(x+\psi_{A}\right)}-\mathrm{e}^{j\left(x+\psi_{A}\right)} \mathrm{e}^{-j 4 x} d x\right.
$$

После интегрирования и соответствующих подстановок получим

$$
z_{A 4}=V_{A} F^{3}\left(F^{2} P_{A}\left(H_{A}^{5}-a H_{B}^{5}\right) / 5-\bar{P}_{A}\left(H_{A}^{3}-H_{B}^{3}\right) / 3\right) / \pi .
$$

По таблице 


$$
z_{4}=z_{A 4}+a^{2} z_{B 4}+a z_{C 4},
$$

откуда для первого приближения

$$
z_{4}=3 F^{3}\left(F^{2}(1-a) U_{A 0} / 5+\left(F^{2}\left(1-a^{2}\right)+a-1\right) V H_{A 0}\right) / \pi,
$$

где

$$
\begin{aligned}
& U_{A 0}=\left(U_{A}+U_{B}+U_{C}\right) / 3 \approx\left(\Delta_{A}+a^{2} \Delta_{B}+a \Delta_{C}+j\left(\psi_{A}+a^{2} \psi_{B}+a \psi_{C}\right)\right) / 3, \\
& H_{A 0}=\left(H_{A}+a^{2} H_{B}+a H_{C}\right) / 3 \approx-j\left(\chi_{A}+a^{2} \chi_{B}+a \chi_{C}\right) / 3 .
\end{aligned}
$$

При отсутствии нулевого проводника $U_{A 0}=0$ и тогда

$$
z_{4}=3 F^{3}\left(F^{2}\left(1-a^{2}\right)+a-1\right) V H_{A 0} / \pi .
$$

Как видно, уровень четвертой гармоники полностью обусловлен характером схемы управления тиристорами. Учитывая величину модуля коэффициента перед $H_{A 0}$, который стремится к нулю при уменьшении угла управления до нуля, можем сказать, что уровень четвертой гармоники понижается с уменьшением угла управления и будет незначительным при максимальном токе.

Для получения окончательных результатов необходимо выяснить одно свойство асимметричной системы включения тиристоров. В случае нашей модели угол отпирания тиристора характеризуется комплексным числом, модуль которого равен единице, а фаза равна соответствующему углу отклонения от симметричного положения.

Рассмотрим подробнее случай малых отклонений

$$
H_{A} \approx 1-i \chi_{A}, \quad H_{B} \approx 1-j \chi_{B}, \quad H_{C} \approx 1-j \chi_{C} .
$$

Для $H_{A 0}$ и $H_{A 2}$ получим

$$
\begin{aligned}
& H_{A 0}=\sqrt{3}\left(\left(\chi_{B}-\chi_{C}\right)+2 j\left(\chi_{A}-0,5 \chi_{B}-0,5 \chi_{C}\right)\right) / 6, \\
& H_{A 2}=-\sqrt{3}\left(\left(\chi_{B}-\chi_{C}\right)+2 j\left(\chi_{A}-0,5 \chi_{B}-0,5 \chi_{C}\right)\right) / 6,
\end{aligned}
$$

откуда видно, что

$$
\left|H_{A 0}\right|=\left|H_{A 2}\right|
$$

Следовательно, $H_{A 0}$ и $H_{A 2}$ - зависимые переменные и реализовать любые заданные их конфигурации путем выбора $\chi_{A}, \chi_{B}$ и $\chi_{C}$ в общем случае одновременно не удается. Докажем теперь следующее

У тверждение. Подавление второй гармоники по формуле приводит к повышению частоты данной пульсации в два раза.

Д ок аз а тельство. Так как $z_{2}$ является линейной комбинацией $U_{A 2}$ и $H_{A 2}$, то $z_{2}$ равняется нулю, если $U_{A 2}$ и $H_{A 2}$ генерируют свои доли с одинаковой амплитудой и в противофазе. Так как $\left|H_{A 2}\right|=$ $=\left|H_{A 0}\right|$ и множители при $H_{A 2}$ и $H_{A 0}$ в формулах (10) и (17) одинаковы (по модулю), то

$$
\left.\left|z_{4}\right|\right|_{H_{A 2}=\left(\left(F^{2}+a\right)^{-1} U_{A 3}\right) / V}=\left.\left|z_{2}\right|\right|_{H_{A z}=0} .
$$




\section{Выводы}

1. Қаждая низкочастотная гармоника в первом приближении линейно связана с соответствующей симметричной составляющей сети и тиристоров.

2. Для подавления второй гармоники, вызванной асимметрией сети, $\chi_{A}$, $\chi_{B}$ и $\chi_{c}$ следует выбирать так, чтобы удовлетворялось выражение (13).

3. Подавление второй гармоники не реализуется при малых углах управления.

4. Подавление второй гармоники (по формуле (13)) приводит к повышению частоты данной пульсации вдвое.

\section{ЛИТЕРАТ У РА}

1. Е мельянов А. В., В кн.: Преобровательные устройства и системы возбуждения синхронных машин, Л., «Наука», 1973, с. 29-39.

2. Булга ков К. В., Лоскутов Е. Д., Изв. ВУЗов, Электромеханика, № 8, 861865 (1970).

3. Попо в С. Г., Электричество, № 6, 69-71 (1979).

4. Р а бине р Л., Гоулд Б., Теория и применение цифровой обработки сигналов, М., «Мир», 1978.

5. Лоскутов Е. Д., Поли бин В. П., Торговицкий И. М., Способ подавления низкочастотных гармоник пульсаций выпрямленного тока, Авт. свид. СССР № 230269, Бюл. изобр. № 34 (1968).

6. С акович А., Мамсуров А., Н в а А., Изв. АН ЭССР, Сер. физ.-матем. и техн. наук, Х, № 3, с. 400-407 (1966).
Ннститут термофизики и электрофизики Академии наук Эстонской ССР
Поступила в редакцию 2/VII 1980

\section{P. TERASMAA}

\section{KOLMEFAASILISE TUURITAVA ALALDI VALJUNDPINGE MADALSAGEDUSLIKE KOMPONENTIDE KORVALDAMINE TURISTORIDE JUHTIMISE SEADUSE ABIL}

Artiklis on esitatud lihtsad seosed teise ja neljanda harmoonilise arvutamiseks esimeses lähenduses (pōhisageduseks loetakse vōrgusagedust). On tuletatud seos türistoride juhtimiseks, mille korral ebasümmeetriline võrk ei pōhjusta teise harmoonilise genereerimist, ja tôestatud, et sel juhul vastava pulsatsiooni sagedus kahekordistub.

\section{P. TERASMAA}

\section{ELIMINATION OF LOW FREQUENCY HARMONICS IN THE OUTPUT VOLTAGE OF A THREE-PHASE BRIDGE RECTIFIER BY THE CONTROL OF THYRISTOR FIRING ANGLES}

The present paper deals with the formation of low frequency harmonics with a threephase bridge operating on asymmetrical network. For the sake of simplification thyristors are considered ideal and loads active-inductive. 
The relations are given in terms of the symmetrical components of source voltage and firing angle system.

Simple relation for the second and fourth harmonic (the first is the frequency of network voltage) are obtained owing to the use of linearity and shifting properties of the Fourier transform and due to linearization around the symmetrical position.

The following theses are verified by above simplifications:

1. To a first approximation, every low frequency harmonic is related to the corresponding symmetrical component of the network and firing angles.

2. Compensation of the second harmonic is impossible for minor cut-off angles.

3. Compensation of the second harmonic leads to twofold increase in the given pulsation frequency. 